\title{
Pathologic Process
}

National Cancer Institute

\section{Source}

National Cancer Institute. Pathologic Process. NCI Thesaurus. Code C16956.

A biologic function or a process having an abnormal or deleterious effect at the subcellular, cellular, multicellular, or organismal level. 